\title{
28 Research Square \\ Genome study reveals links between gut microbiome and complex diseases
}

Fengzhe Xu

Yuanqing Fu

Ting-yu Sun

Zengliang Jiang

Zelei Miao

Menglei Shuai

Wanglong Gou

Chu-wen Ling

Jian Yang

Jun Wang

Yu-ming Chen

Ju-Sheng Zheng

\section{Video Byte}

Keywords: Microbiome, gut microbiome, host genetics, bidirectional Mendelian randomization analyses, disease-microbiome features, complex human disease, genome-wide association study, GWAS, East Asia, China, alpha diversity, heritability, enterotype, polygenic score, genetic variant, single-nucleotide polymorphism, SNP, beta diversity, disease etiology, Guangzhou Nutrition and Health Study

Posted Date: November 12th, 2020

DOI: https://doi.org/10.21203/rs.3.rs-107416/v1

License: (9) This work is licensed under a Creative Commons Attribution 4.0 International License. Read Full License 


\section{Abstract}

Growing evidence suggests a link between the gut microbiome and physiological processes, including the immune response and neurological function. But the connection between the gut microbiome and complex diseases remains largely unexplored. A new study suggests that, at least among East Asian populations, distinct microbiome features could give rise to certain diseases. Researchers performed a microbiome genome-wide association study using samples from 1,475 Chinese participants. Their goal was to identify genetic variants among the participants associated with the gut microbiome. According to their findings, certain bacteria could be linked to the risk of certain diseases. For example, developing an irregular heartbeat is associated with an increased abundance of Burkholderiales and Alcaligenaceae, and decreased abundance of Lachnobacterium, Bacteroides coprophilus and Barnesiellaceae. And microbiome features could be clustered by disease, including different cancers, Alzheimer's disease, and hepatitis. Larger studies across diverse populations could refine the causal relationships revealed by the current study and perhaps offer vital clues on how to make existing disease-fighting drugs more effective. 